\title{
ALMOST ISOLATED SPECTRAL PARTS AND INVARIANT SUBSPACES $\left({ }^{1}\right)$
}

\author{
BY
}

C. R. PUTNAM

\begin{abstract}
Let $T$ be an operator with spectrum $\sigma(T)$ on a Hilbert space. A compact subset $E$ of $\sigma(T)$ is called a disconnected part of $\sigma(T)$ if, for some bounded open set $A, E$ is the closure of $\sigma(T) \cap A$ and $\sigma(T)-E$ is the union of the isolated parts of $\sigma(T)$ lying completely outside the closure of $A$. The set $E$ is called an almost isolated part of $\sigma(T)$ if, in addition, the set $A$ can be chosen so as to have a rectifiable boundary $\partial A$ on which the subset $\sigma(T) \cap \partial A$ has arc length measure 0 . The following results are obtained. If $T$ is subnormal and if $E$ is a disconnected part of $\sigma(T)$ then there exists a reducing subspace $T$ of $T$ for which $\sigma(T \mid \mathscr{R})=E$. If $T^{*}$ is hyponormal and if $E$ is an almost isolated part of $\sigma(T)$ then there exists an invariant subspace of $T$ for which $\sigma(T \mid W)=E$. An example is given showing that if $T$ is arbitrary then an almost isolated part of $\sigma(T)$ need not be the spectrum of the restriction of $T$ to any invariant subspace.
\end{abstract}

1. Introduction. Let $T$ denote a bounded operator on a Hilbert space $\mathfrak{Z}$ with spectrum $\sigma(T)$ and resolvent set $\rho(T)$. A bounded open set $D$ of the complex plane is called an admissible domain of $T$ (cf. Riesz and Sz.-Nagy [14, p. 418]) if its boundary $\partial D$ consists of a finite number of rectifiable closed curves lying in $\rho(T)$ and positively oriented with respect to $D$. A compact subset $\sigma$ of $\sigma(T)$ is called an isolated part of $\sigma(T)$ if its distance from $\sigma(T)-\sigma$ is positive, in which case there exists some admissible domain $D$ for which

$$
\sigma=\sigma(T) \cap D .
$$

Moreover, if $\sigma$ is nonempty then the Riesz integral

$$
P_{\sigma}=-(2 \pi i)^{-1} \int_{\partial D}(T-z)^{-1} d z
$$

defines a projection $\left(P_{\sigma}^{2}=P_{\sigma}\right)$ for which the space $\mathbb{R}_{\sigma}=P_{\sigma}\left(\mathfrak{F}_{2}\right)$ is invariant under $T$ and $\sigma\left(T \mid \mathbb{R}_{\sigma}\right)=\sigma$. In fact, $\mathbb{R}_{\sigma}$ is a hyperinvariant subspace of $T$, that is, $\mathbb{R}_{\sigma}$ is

Received by the editors June 26, 1974.

AMS (MOS) subject classifications (1970). Primary 47A15, 47A10; Secondary 47B20, 47B47.

Key words and phrases. Isolated part of spectrum, disconnected part of spectrum, almost isolated part of spectrum, invariant subspaces, subnormal operators, hyponormal operators.

(1) This work was supported by National Science Foundation research grant $6460-50-13955$. 
invariant under any bounded operator which commutes with $T$.

A compact set $E$ of $\sigma(T)$ will be called a disconnected part of $\sigma(T)$ if there exists a bounded open set $A$ such that

$$
E=(\sigma(T) \cap A)^{-} \text {and } \sigma(T)-E=\bigcup\left\{\sigma: \sigma \subset \mathbf{C}-A^{-}\right\},
$$

where the union is taken over all isolated parts of $\sigma(T)$ which lie completely outside the closure, $A^{-}$, of $A$. Such a set $E$ will be called an almost isolated part (a.i.p.) of $\sigma(T)$ if, in addition, the set $A$ can be chosen so that $\partial A$ consists of a finite number of rectifiable closed curves oriented positively with respect to $A$ and satisfying

$$
\operatorname{meas}_{1}(\sigma(T) \cap \partial A)=0,
$$

where the measure denotes arc length on $\partial A$. Clearly, an isolated part $E$ of $\sigma(T)$ is also an a.i.p. of $\sigma(T)$, while the converse holds if and only if the set $A$ satisfying (1.3) and (1.4) can be chosen so that also $\sigma(T) \cap \partial A=\varnothing$. Note that if, for instance,

$$
\sigma(T)=\{z:|z+1| \leqslant 1\} \cup\{1,1 / 2,1 / 3, \ldots\},
$$

then $E=\{z:|z+1| \leqslant 1\}$ is an a.i.p. of $\sigma(T)$ but not an isolated part. Similarly, $[-1,0]$ is an a.i.p. of $\sigma(T)=[-1,0] \cup\{1,1 / 2,1 / 3, \ldots\}$; on the other hand, $\{0\}$ is not an a.i.p. of $\sigma(T)=\{0\} \cup\{1,1 / 2,1 / 3, \ldots\}$.

Obviously, a set $E$ is a disconnected part or an almost isolated part of $\sigma(T)$ if and only if the set $\{\bar{z}: z \in E\}$ bears the corresponding relation to $\sigma\left(T^{*}\right)$.

In general, an a.i.p. of $\sigma(T)$ need not be the spectrum of any restriction of $T$ to an invariant subspace. In fact, it will be shown $(\S 5)$ that there exists an operator $T$ for which (1.5) holds and such that if $\mathfrak{M}$ is any nontrivial invariant subspace of $T$ then

$$
\sigma(T \mid M) \cap\{1,1 / 2,1 / 3, \ldots\} \neq \varnothing
$$

We recall some definitions and properties. An operator $T$ is said to be hyponormal if $T^{*} T-T T^{*} \geqslant 0$, in which case it is known (Stampfli [16]) that

$$
\|(T-z) x\| \geqslant\left\|\left(T^{*}-\bar{z}\right) x\right\| \geqslant[\operatorname{dist}(z, \sigma(T))]\|x\| .
$$

The restriction of a hyponormal operator to an invariant subspace is also hyponormal; see Berberian [1, p. 161]. An operator $T$ is said to be subnormal if it has a normal extension on a larger Hilbert space. The subnormal operators form a proper subset of the hyponormal operators; see, e.g., Halmos [6, p. 105].

A hyponormal operator is called completely hyponormal if it has no normal part, that is, if there does not exist a (nontrivial) reducing subspace of $T$ on which it is normal. Similarly, one can define a completely subnormal operator. It is 
known that if $T$ is completely hyponormal and if $D$ is any open disk for which $\sigma(T) \cap D$ is nonempty then $\sigma(T) \cap D$ must have positive planar measure (Putnam [9]). Conversely, if $\alpha$ is any compact set with the property that for any open disk $D$ the set $\alpha \cap D$ has positive planar measure whenever it is nonempty, then $\alpha$ is the spectrum of some completely hyponormal operator; see Putnam [12]. In Carey and Pincus [4] it is shown that, in fact, the operator $T$ can be chosen so that $T^{*} T-T T^{*}$ even has rank 1 . For necessary and sufficient conditions that a compact set be the spectrum of a completely subnormal operator, see Clancey and Putnam [2].

For a summary of properties of certain "almost normal" operators, see Putnam [11].

Let $T$ be normal and let $\alpha$ denote any (nonempty) compact subset of $\sigma(T)$ of the form $\alpha=(\sigma(T) \cap A)^{-}$where $A$ is an open set. Then it is easy to see that there exists an invariant subspace $\mathbb{R}$ of $T$ for which $\sigma(T \mid \mathscr{R})=\alpha$. In fact, if $E_{z}$ is the spectral family of $T$ then $\mathbb{R}=E(\alpha) \mathbb{S}_{2}$ has the required property. That no such property can hold for hyponormal or even subnormal operators is clear. In fact, one need only consider a completely subnormal isometry, say,

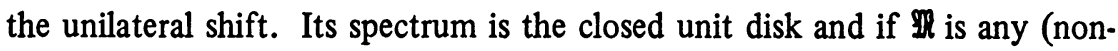
trivial) invariant subspace then the restriction of this shift to $\mathbb{R}$ is also a completely subnormal isometry with the same spectrum. It is interesting though to note that if $T$ is an arbitrary contraction $(\|T\| \leqslant 1)$ on a Hilbert space $\mathscr{5}$, then there exists an isometry $V$ on a larger Hilbert space such that $T$ is the restriction of $V^{*}$ to $\mathscr{F}_{\text {; }}$ see Sz.-Nagy and Foias $[19$, p. 11]. In particular, any compact subset of the closed unit disk is the spectrum of some coisometry restricted to an invariant subspace. See also Sz.-Nagy and Foias [19, pp. 66ff] and Douglas, Muhly and Pearcy [5].

2. Subnormal operators. Let $T$ be subnormal and suppose that $\sigma_{1}$ and $\sigma_{2}=\sigma(T)-\sigma_{1}$ are nonempty isolated parts of $\sigma(T)$. As already noted, there exist invariant subspaces $\mathbb{P}_{k}=P_{k}\left(\mathcal{F}_{2}\right) \quad\left(P_{k}=P_{\sigma_{k}}\right.$ of (1.2)) for which $\sigma\left(T \mid \mathbb{R}_{k}\right)=$ $\sigma_{k}(k=1$ and 2$)$. Since $T$ is subnormal, much more can be said however. In fact, $P_{k}$ is selfadjoint, $\mathbb{P}_{k}$ reduces $T, T \mid \mathbb{P}_{k}$ is subnormal and

$$
T=T\left|\mathbb{R}_{1} \oplus T\right| \mathbb{R}_{2}
$$

see Williams [20, pp. 97-98]. These facts can be used to establish

THEOREM 1. Let $T$ be subnormal and let $E(\neq \varnothing, \sigma(T))$ be a disconnected part of $\sigma(T)$. Then there exist subspaces $\mathfrak{X}_{k}(k=1,2)$, which are hyperinvariant and reducing for $T$, and for which $T \mid \mathbb{M}_{k}$ is subnormal, $\sigma\left(T \mid \mathbb{R}_{1}\right)=E$ and $\sigma\left(T \mid \mathbb{R}_{2}\right)$ $=(\sigma(T)-E)^{-}$, and $(2.1)$ holds. 
Proof. According to the definition of a disconnected part of $\sigma(T)$, there exists a bounded open set $A$ satisfying condition (1.3). Clearly, $\sigma(T)-E=$ $\bigcup_{\sigma_{k}}$ where $\sigma_{1}, \sigma_{2}, \ldots$ is a (possibly finite) sequence of pairwise disjoint isolated parts of $\sigma(T)$. Then the operators $P_{k}=P_{\sigma_{k}}$ are selfadjoint and pairwise orthogonal, the spaces $\mathbb{R}_{k}=P_{k}\left(\mathscr{S}_{2}\right)$ are hyperinvariant, reducing subspaces of $T$, and the operators $T \mid \mathcal{R}_{k}$ are subnormal. If $Q=\Sigma \bigoplus P_{k}$ and $R=I-Q$, then $Q$ and $R$ are orthogonal (selfadjoint) projections, $\mathbb{P}_{1}=R\left(\mathscr{F}_{2}\right)$ and $\mathbb{M}_{2}=Q(5)$ are hyperinvariant, reducing subspaces of $T$, and (2.1) holds, where both summands are subnormal. Since $T \mid \mathbb{R}_{2}=\Sigma \bigoplus\left(T \mid P_{k}(\mathscr{L})\right)$ and $\sigma\left(T \mid P_{k}(\mathscr{L})\right)=\sigma_{k}$, then $(\sigma(T)-E)^{-} \subset \sigma\left(T \mid \mathbb{R}_{2}\right)$. The reverse inclusion, hence equality, follows from (1.7). That $\sigma\left(T \mid \mathbb{M}_{1}\right) \supset E$ is clear from (1.3) and (2.1). If $R_{n}=I-\left(P_{1}+\ldots\right.$ $+P_{n}$ ) then (cf. the beginning of $\left.\S 1\right) \sigma\left(T \mid R_{n}(\delta)\right)=\sigma(T)-\left(\sigma_{1} \cup \ldots \cup \sigma_{n}\right)$. Since $R_{n} \rightarrow R$ (strongly), another appeal to (1.7) shows that $\sigma\left(T \mid \mathbb{M}_{1}\right)=E$. This completes the proof of Theorem 1 .

3. Hyponormal operators. It is interesting to contrast the above situation for subnormal operators with the corresponding one for hyponormal operators. As was indicated above, if $T$ is subnormal and if $\sigma(T)$ is the union of two nonempty disjoint compact sets (hence, each is an isolated part of $\sigma(T)$ ), then $T$ is reducible. In particular, if $T^{*} T-T T^{*}$ has rank 1 , then $T$ has a normal part. On the other hand, it is possible that $T$ is completely hyponormal, $\sigma(T)$ is the union of disjoint, nonempty isolated parts $\sigma_{1}$ and $\sigma_{2}$, but $T$ is irreducible. A simple example is furnished by the singular integral operator $T=H+i J$ on $\mathscr{F}=L^{2}(\alpha)$, where $\alpha=[0,1 / 4] \cup[3 / 4,1]$ and where $H$ and $J$ are the selfadjoint operators

$$
(H x)(t)=t x(t), \quad(J x)(t)=-(i \pi)^{-1} \int_{\alpha}(s-t)^{-1} x(s) d s .
$$

(Here, the integral operator is interpreted as a Cauchy principal value integral.) It is easy to verify that $T$ is hyponormal and that $T^{*} T-T T^{*}$ has rank 1 . In addition, it turns out that $\sigma(T)$ consists of the two isolated parts $\sigma_{1}=[0,1 / 4] \times$ $[-1,1]$ and $\sigma_{2}=[3 / 4,1] \times[-1,1]$, and that, further, $T$ is irreducible; see Clancey and Putnam [3, p. 452].

Let $T$ be hyponormal and suppose that $\sigma$ is a nonempty isolated part of $\sigma(T)$. Then, as noted above, there exists an invariant subspace $\Re$ of $T$ for which $(T \mid \mathcal{M}$ is hyponormal and) $\sigma(T \mid \mathfrak{R})=\sigma$. Whether a similar assertion concerning the spectrum holds if $\sigma$ is replaced by an almost isolated part $E$ will, in general, remain open. However, the following will be proved.

THEOREM 2. Let $T^{*}$ be hyponormal, and let $E$ be an almost isolated part of $\sigma(T)$ contained in an associated bounded open set $A$ considered in $\S 1$ and satisfying (1.3) and (1.4). Then there exists a hyperinvariant subspace $\Re$ of $T$ for 
which

$$
\sigma(T \mid \mathbb{R})=E \text {. }
$$

REMARKS. We do not know whether the assertion of Theorem 2 is still valid if, for instance, either the hypothesis (1.4) is omitted (so that $E$ is assumed only to be a disconnected part of $\sigma(T)$ ) or if the hypothesis that $T^{*}$ be hyponormal is replaced by the hypothesis that $T$ be hyponormal. Of course, if $T$ is hyponormal and if $E$ is an almost isolated part of $\sigma(T)$, then Theorem 2 asserts the existence of a hyperinvariant subspace $\Re$ of $T^{*}$ for which $\sigma\left(T^{*} \mid \Re\right)=$ $\{\bar{z}: z \in E\}$. It is noteworthy also that if $T$ is hyponormal and if $E$ is a disconnected part of $\sigma(T)$ for which $E \neq \sigma(T)$, then there exists a hyperinvariant subspace $\mathfrak{M}$ of $T$ for which $\sigma(T \mid \mathfrak{M})=(\sigma(T)-E)^{-}$. This can be deduced from a result of Stampfli [18] (cf. Theorem 2 there). Of course, if $T$ is subnormal, even more can be claimed (cf. Theorem 1 above).

A related problem concerns the existence of invariant subspaces of operators $T$ for which $\sigma(T)$ is connected but is such that $\sigma(T)=E \cup F$, where $E$ and $F$ are compact and $E \cap F$ has linear (arc length) measure 0 as a subset of a rectifiable curve. In this connection, see Lautzenheiser [7] and Putnam [13], where the operators considered are subnormal, and Stampfli [17], where the operators satisfy certain resolvent growth conditions, and certain restrictions are also imposed on the degree of contact between $E$ and $F$. In [17], one can also find numerous references.

Before beginning the proof of Theorem 2, it will be convenient to recall some results obtained in Putnam [10]. Let $T^{*}$ be hyponormal so that

$$
T T^{*}-T^{*} T=D \geqslant 0 .
$$

If $D$ has the spectral family $G_{t}$ and if $x=G(s, \infty) x$, where $s>0$, then for each vector $y$ in $\mathcal{S}_{2}$, the function $F(z)=\left((T-z)^{-1} x, y\right)$ is, in addition to being analytic for $z \in \rho(T)$, also continuous and bounded in $C-\Pi$, where $\Pi$ is the set of complex $z$ for which either $z$ is in the point spectrum of $T$ or $\bar{z}$ is in the point spectrum of $T^{*}$. (The set $P$ occurring in Putnam [10, p. 165], also in $[11$, p. 622], should be defined as $\Pi$ above.) By an adaptation of that argument we can obtain other vectors $x$ for which $(T-z)^{-1} x$ is (weakly) continuous and bounded, as above, at least for certain parts of the plane including portions of $\sigma(T)$.

To see this, let $T$ have the rectangular representation $T=H+i J$, where $H$ has the spectral resolution $H=\int \lambda d E_{\lambda}$. Then, if $z=t+i$, one has by (3.2),

$$
(T-z)(T-z)^{*}=(H-t)^{2}+(J-s)^{2}+1 / 2 D \geqslant(H-t)^{2} .
$$

An argument like that in $[10$, p. 166], shows that if $\alpha$ is any Borel set of the 
real line and if $z$ is not in $\Pi$ and $t$ is not in $\alpha^{-}$then $\left\|(T-z)^{-1} x\right\|^{2} \leqslant$ $\int_{\alpha}(\lambda-t)^{2} d\left\|E_{\lambda} x\right\|^{2}$. (Incidentally, if $t$ is not in the point spectrum of $H$, this last inequality holds for arbitrary Borel sets $\alpha$ provided $x$ is in the domain of $(H-t)^{-1}$. This situation surely obtains if $T$ is completely hyponormal, in which case $H$ is even absolutely continuous; cf. [8, p. 43].) Hence,

$$
\left\|(T-z)^{-1} E(\alpha) x\right\| \leqslant[\operatorname{dist}(\operatorname{Re}(z), \alpha)]^{-1}\|E(\alpha) x\|, \quad z \notin \Pi \text { and } \operatorname{Re}(z) \notin \alpha^{-} .
$$

An argument similar to that of $[10$, p. 166] shows that

$$
\begin{aligned}
& (T-z)^{-1} E(\alpha) x \text { is (weakly) continuous in } \\
& {\left[\rho(T) \cup\left\{z: \operatorname{Re}(z) \text { outside } \alpha^{-}\right\}\right]-\Pi \text {. }}
\end{aligned}
$$

4. Proof of Theorem 2. Let $Z=\sigma(T) \cap \partial A$, so that, by (1.4), $Z$ is a closed subset of $\partial A$ of (arc length) measure 0 . Let $L$ denote the linear manifold of vectors $x$ for which $\left\|(T-z)^{-1} x\right\|$ is bounded and $(T-z)^{-1} x$ is weakly continuous on $\partial A-Z$. Then one may define the "projection"

$$
P x=-(2 \pi i)^{-1} \int_{\partial A}(T-z)^{-1} x d z, \quad x \in L,
$$

where, in fact, $(P x, y)$ exists as a Riemann integral for $x$ in $L$ and $y$ in $\mathcal{F}_{\text {. Fur- }}$ ther, it is clear that $L$ is invariant under $T$. It will first be shown that if $z_{0} \in$ $\sigma(T) \cap A$ then there exist vectors $x_{n} \in L \quad(n=1,2, \ldots)$ satisfying

$$
\left(T-z_{0}\right) P x_{n} \rightarrow 0, \quad\left\|P x_{n}\right\|=1 \quad\left(x_{n} \in L\right) .
$$

To see this, note that if $z_{0}(\in \sigma(T) \cap A)$ is in the point spectrum of $T$ then $\left(T-z_{0}\right) x=0$ and hence $(T-z)^{-1} x=\left(z_{0}-z\right)^{-1} x$ for some unit vector $x$ and for $z \in \rho(T)$. Clearly, $x \in L$ and $P x=x$, so that (4.2) holds with all $x_{n}=x$. Further, if there exists a sequence $\left\{z_{n}\right\}$ such that $z_{n} \rightarrow z_{0}, z_{n} \in$ $\sigma(T) \cap A$, and each $z_{n}$ belongs to the point spectrum of $T$, then $\left(T-z_{n}\right) u_{n}=$ 0 , where $\left\|u_{n}\right\|=1$. Since $(T-z)^{-1} u_{n}=\left(z_{n}-z\right)^{-1} u_{n}$ for $z \in \rho(T)$, and since $z_{n} \in A$, then $u_{n} \in L$ and $\left(T-z_{n}\right) P u_{n}=0$ with $P u_{n}=u_{n}$. Clearly, (4.2) holds with $x_{n}=u_{n}$.

Suppose then that $z_{0} \in \sigma(T) \cap A$ and that some neighborhood of $z_{0}$ is free of the point spectrum of $T$. It can be supposed that $T^{*}$ is completely hyponormal. (In fact, if $T^{*}$ [hence $T$ ] has a normal part, this part can be treated separately. As noted earlier, if $N$ is normal and if $D$ is any open set such that $\sigma(N) \cap D \neq \varnothing$, then $N$ has a reducing space on which its spectrum is $(\sigma(N) \cap D)^{-}$.) In view of (1.4), it is easy to see that for almost all real $t$ the line $\{w: \operatorname{Re}(w)=t\}$ does not intersect the set $Z$. Further, since $T^{*}$ is completely hyponormal, then, as noted above, any open disk centered at $z_{0}$ must intersect $\sigma(T)$ in a set of positive planar measure. It follows that there exist $\left\{z_{n}\right\}$ (n= $1,2, \ldots$ ) for which $z_{n} \rightarrow z_{0}, z_{n} \in \sigma(T) \cap A$ but no $z_{n}$ is in the point spectrum 
of $T$, and for which $\left\{w: \operatorname{Re}(w)=\operatorname{Re}\left(z_{n}\right)\right\} \cap Z=\varnothing$. If $n$ is fixed, it is clear that there exists an open interval $\alpha$ (depending on $n$ ) which contains $\operatorname{Re}\left(z_{n}\right)$ and satisfies

$$
\left\{w: \operatorname{Re}(w) \in \alpha^{-}\right\} \cap Z=\varnothing .
$$

It follows from (3.4) and (3.5) that

$$
\begin{aligned}
& \text { if } x=E(\alpha) x \text {, then }(T-z)^{-1} x \text { is weakly continuous } \\
& \text { and }\left\|(T-z)^{-1} x\right\| \leqslant(\text { const) }\|x\| \text { for } z \in \partial A-Z,
\end{aligned}
$$

where "const" depends on $n$ but is independent of $x$ or of $\alpha$, provided that the length of $\alpha$ is sufficiently small. In particular, $x=E(\alpha) x \in L$, so that $P x$ of (4.1) is defined.

Since $T^{*}$ is hyponormal, then $T_{z} T_{z}^{*}-T_{z}^{*} T_{z} \geqslant 0$, where $T_{z}=T-z$ and $z$ is arbitrary. Since $z_{n} \in \sigma(T)$ and $z_{n}$ is not in the point spectrum of $T$, it follows that, for each fixed $n$, there exists a sequence of unit vectors $\left\{u_{k}\right\}$ for which

$$
\text { both }\left(T^{*}-\bar{z}_{n}\right) u_{k} \rightarrow 0 \text { and }\left(T-z_{n}\right) u_{k} \rightarrow 0 \text { as } k \rightarrow \infty \text {. }
$$

If $z=z_{n}$ in (3.3), it is seen that $\left(H-t_{n}\right) u_{k} \rightarrow 0\left(t_{n}=\operatorname{Re}\left(z_{n}\right)\right)$ and consequently, for any fixed (sufficiently small) open interval $\alpha$ containing $t_{n}, u_{k}$ $E(\alpha) u_{k} \rightarrow 0$. Hence, it can be assumed that (4.5) holds with

$$
u_{k}=E\left(\alpha_{k}\right) u_{k}, \quad\left\|u_{k}\right\|=1 \quad(k=1,2, \ldots), u_{k} \in L,
$$

where $\alpha_{k}$ is an open subinterval of $\alpha$ containing $t_{n}$, relation (4.3) holds with $\alpha$ replaced by $\alpha_{k}$, and where $\left|\alpha_{k}\right| \rightarrow 0$ as $k \rightarrow \infty$.

Now, $(T-z) u_{k}=y_{k}+\left(z_{n}-z\right) u_{k}$, where $y_{k}=\left(T-z_{n}\right) u_{k} \rightarrow 0$ as $k \rightarrow \infty$ ( $n$ fixed) and hence, since $z_{n} \in A$,

$$
\begin{aligned}
u_{k} & =(2 \pi i)^{-1} \int_{\partial A}\left(z-z_{n}\right)^{-1} u_{k} d z \\
& =P u_{k}+(2 \pi i)^{-1} \int_{\partial A}\left(z-z_{n}\right)^{-1}(T-z)^{-1} y_{k} d z .
\end{aligned}
$$

For $z \in \partial A-Z,(T-z)^{-1} y_{k}=u_{k}+\left(z-z_{n}\right)(T-z)^{-1} u_{k}$, so that

$$
\left\|(T-z)^{-1} y_{k}\right\| \leqslant\left\|u_{k}\right\|+\left|z-z_{n}\right|\left\|(T-z)^{-1} u_{k}\right\|
$$

and hence, by (4.4) with $x=u_{k}$ of (4.6),

$$
\left\|\left(z-z_{n}\right)^{-1}(T-z)^{-1} y_{k}\right\| \leqslant \text { const for } z \in \partial A-Z,
$$

where "const" is independent of $k$ (for $\left|\alpha_{k}\right|$ sufficiently small). Since $y_{k} \rightarrow 0$ as $k \rightarrow \infty$,

$$
\left\|\left(z-z_{n}\right)^{-1}(T-z)^{-1} y_{k}\right\| \rightarrow 0 \text { as } k \rightarrow \infty \text { for } z \in \partial A-Z .
$$


By (4.7),

$$
\left\|u_{k}-P u_{k}\right\| \leqslant(2 \pi)^{-1} \int_{\partial A}\left\|\left(z-z_{n}\right)^{-1}(T-z)^{-1} y_{k}\right\||d z| .
$$

Hence, by (4.8), (4.9) and Lebesgue's uniformly bounded convergence theorem, $u_{k}-P u_{k} \rightarrow 0$ as $k \rightarrow \infty$. Thus, by (4.5), one can suppose that $\left(T-z_{n}\right) P u_{k}$ $\rightarrow 0$ as $k \rightarrow \infty$ (n fixed), where $u_{k}=u_{k}^{(n)} \in L$ and $\left\|P u_{k}\right\|=1$. Since $z_{n} \rightarrow z_{0}$ as $n \rightarrow \infty$, it is clear that there exists a subsequence $\left\{x_{n}\right\}$ of $\left\{u_{k}^{(n)}\right\}$ satisfying (4.2).

Next, let $M$ denote the closure of the linear manifold of vectors $\{P x\}$ where $x \in L$. Clearly, $\mathbb{R}$ is a hyperinvariant subspace of $T$. Since $z_{0}$ is an arbitrary point of $\sigma(T) \cap A$, it follows from (4.2) that $E \subset \sigma(T \mid M)$. Since $\Re$ is hyperinvariant for $T$ then, in particular, $\mathbb{M}$ is invariant under $(T-z)^{-1}$ whenever $z \in$ $\rho(T)$, and hence $\sigma(T \mid \Re) \subset \sigma(T)$. The proof of (3.1) and hence of Theorem 2 will be completed by showing that

$$
\sigma(T)-E \subset \rho(T \mid \mathbb{R}) .
$$

In order to prove (4.10), let $z_{1} \in \sigma(T)-E$, so that $z_{1} \in \sigma$ where $\sigma$ is some isolated part of $\sigma(T)$ contained in $\mathrm{C}-A^{-}$. Let $D$ denote an associated admissible domain satisfying (1.1) and such that $\partial D$ lies in $\mathbf{C}-A^{-}$. Next, let $x \in L$ and consider the vector function

$$
F(z)=-(2 \pi i)^{-1} \int_{\partial A}(t-z)^{-1}(T-t)^{-1} x d t .
$$

Clearly, $F(z)$ is analytic in $\mathbf{C}-A^{-}$and hence, by the Cauchy integral formula,

$$
F\left(z_{1}\right)=(2 \pi i)^{-1} \int_{\partial D}\left(z-z_{1}\right)^{-1} F(z) d z .
$$

Also, $(T-z) F(z)=P x$ for $z \in \mathrm{C}-A^{-}$. If $Q$ is defined by

$$
Q=(2 \pi i)^{-1} \int_{\partial D}\left(z-z_{1}\right)^{-1}(T-z)^{-1} d z,
$$

then $Q$ is clearly a bounded operator on $\mathscr{S}_{2}$ commuting with $T$ and satisfying $Q P x=F\left(z_{1}\right)$. Hence, $Q\left(T-z_{1}\right) P x=\left(T-z_{1}\right) Q P x=P x$, and so, $Q\left(T-z_{1}\right) y=$ $\left(T-z_{1}\right) Q y=y$ for any $y$ in $\mathbb{M}$. Thus $z_{1} \in \rho(T \mid \mathbb{R})$, that is, (4.10). This completes the proof of Theorem 2 .

5. An example. It will be shown that there exists an operator $T$ with spectrum given by (1.5) and with the property that (1.6) holds for every invariant subspace of $T$. First, consider the sequence of nilpotent matrices

$$
A_{1}=0, A_{2}=\left(\begin{array}{ll}
0 & 1 \\
0 & 0
\end{array}\right), A_{3}=\left(\begin{array}{lll}
0 & 1 & 0 \\
0 & 0 & 1 \\
0 & 0 & 0
\end{array}\right), A_{4}=\left(\begin{array}{llll}
0 & 1 & 0 & 0 \\
0 & 0 & 1 & 0 \\
0 & 0 & 0 & 1 \\
0 & 0 & 0 & 0
\end{array}\right), \ldots,
$$


where each $A_{n}$ is regarded as an operator on the corresponding $n$-dimensional Hilbert space $\mathcal{F}_{n}$. Thus, $\sigma\left(A_{n}\right)=0$ for $n=1,2, \ldots$ Let $D=\{z:|z|<1\}$ and, for each $n$, choose a polynomial $p_{n}(z)$ such that

$$
\begin{aligned}
& p_{n}(0)=-1+1 / n, p_{n}(D) \subset\{z:|z| \leqslant 1+1 / n\} \text { and, for each } z \text { in } D, \\
& \text { there exists a } w(=w(z)) \text { in } D \text { satisfying }\left|z .-p_{n}(w)\right|<1 / n .
\end{aligned}
$$

That this can be done can be seen as follows. First, note that $D$ can be mapped conformally onto itself by a function $f_{n}(z)$ for which $f_{n}(0)=-1+1 / n$ and such that the mapping extends to a homeomorphism of $D^{-}$onto itself. (In fact, $f_{n}(z)$ can even be chosen to be a linear fractional transformation.) By Mergelyan's theorem (or less), the extended function $f_{n}(z)$ can be uniformly approximated on $D^{-}$by polynomials, and hence there exists a polynomial $q_{n}(z)$ for which $\left|f_{n}(z)-q_{n}(z)\right|<1 / 2 n$ for $z$ in $D^{-}$; cf. Rudin $\left[15, \mathrm{p}\right.$. 386]. If $c_{n}=$ $-1+1 / n-q_{n}(0)$, then $\left|c_{n}\right|<1 / 2 n$ and so $p_{n}(z)=q_{n}(z)+c_{n}$ satisfies (5.1).

Next, note that $\left\|A_{n}\right\|=1$ for all $n$ and hence, by (5.1) and the von Neumann-Heinz theorem (cf. Halmos [6, p. 123]),

$$
\left\|p_{n}\left(A_{k}\right)\right\| \leqslant 1+1 / n \quad(n, k \text { arbitrary positive integers), }
$$

where $p_{n}\left(A_{k}\right)$ is regarded as an operator on $\mathscr{F}_{k}$. If

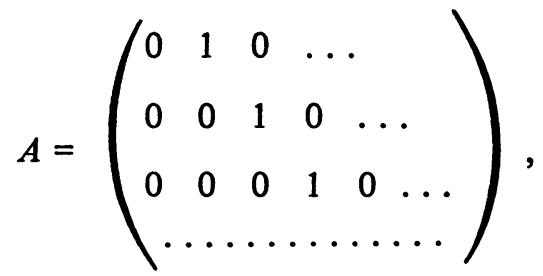

then $A$ is the adjoint of the unilateral shift, $\sigma(A)=D^{-}$and hence $\sigma\left(p_{n}(A)\right)=$ $p_{n}(\sigma(A))=p_{n}\left(D^{-}\right)$; further, every point of $D$ is in the point spectrum of $A$. Next, let $\left\{z_{1}, z_{2}, \ldots\right\}$ be a dense subset of $D$. Clearly, for each fixed $n=$ $1,2, \ldots$, one can choose a positive integer $k_{n}$, together with $n$ unit vectors $x_{n j} \in \mathcal{F}_{k_{n}}(j=1, \ldots, n)$ such that $k_{1}<k_{2}<\ldots$ and

$$
\left\|\left(p_{n}\left(A_{k_{n}}\right)-z_{j}\right) x_{n j}\right\|<2 / n, \quad j=1, \ldots, n .
$$

Let $T=\Sigma_{n=1}^{\infty} \bigoplus B_{n}$ on $\mathcal{F}_{2}=\Sigma_{n=1}^{\infty} \oplus \mathcal{L}_{k_{n}}$, where $B_{n}=(-1+2 / n) I_{k_{n}}-$ $p_{n}\left(A_{k_{n}}\right)$ on $\mathfrak{F}_{k_{n}}$. Since $\sigma\left(A_{k}\right)=\{0\}$ for all $k$, it is clear from $(5.1)$ that $\sigma\left(B_{n}\right)=$ $\{-1+2 / n+1-1 / n\}=\{1 / n\}$. In view of (5.2), $\lim _{\sup _{n \rightarrow \infty}}\left\|B_{n}+I_{k_{n}}\right\| \leqslant 1$ and hence $\sigma(T) \subset\{z:|z+1| \leqslant 1\} \cup\{1,1 / 2,1 / 3, \ldots\}$. This fact combined with (5.3) now yields (1.5).

Finally, it will be shown that if $M(\neq 0)$ is an invariant subspace of $T$ then (1.6) holds. Let $P_{j}(j=1,2, \ldots)$ denote the projection of $\mathscr{L}_{2}=\Sigma \bigoplus \mathcal{S}_{k_{n}}$ 
onto $\mathscr{S}_{k_{j}}$. Clearly, $P_{j} T=T P_{j}$ and $\mathbb{R}_{j}=P_{j} \mathfrak{M}\left(\subset \mathscr{L}_{k_{j}}\right)$ is an invariant subspace of $T$ (and $B_{j}$ ). Since $\mathbb{R} \neq 0$, then $\Re_{p} \neq 0$ for some $p$. Also $T\left|\Re_{p}=B_{p}\right| \mathbb{R}_{p}$ and, since $\partial \sigma\left(B_{p} \mid \mathscr{M}_{p}\right) \subset \sigma\left(B_{p} \mid \mathscr{F}_{c} k_{p}\right)=\{1 / p\}$, it follows that $\sigma\left(B_{p} \mid \mathbb{R}_{p}\right)=\{1 / p\}$ and hence $\sigma\left((T+I) \mid \mathbb{M}_{p}\right)=\{1+1 / p\}$. Consequently, if $S=T+I$,

$$
1+1 / p=\lim _{n \rightarrow \infty}\left\|\left(S \mid \mathbb{R}_{p}\right)^{n}\right\|^{1 / n}=\lim _{n \rightarrow \infty}\left\|\left(P_{p} S \mid \mathbb{R}\right)^{n}\right\|^{1 / n} \leqslant \lim _{n \rightarrow \infty}\left\|(S \mid \mathbb{R})^{n}\right\|^{1 / n},
$$

and so the spectral radius of $S \mid M$ is not less than $1+1 / p$. This means that $\sigma(T \mid \mathfrak{R})$ is not a subset of $\{z:|z+1| \leqslant 1\}$, and, since $\partial \sigma(T \mid \mathfrak{\Re}) \subset \sigma(T)$, relation (1.6) follows.

REMARKS. We wish to acknowledge a helpful correspondence with I. D. Berg, especially in connection with the spectral radius argument of the preceding paragraph.

\section{REFERENCES}

1. S. K. Berberian, Introduction to Hilbert space, Univ. Texts in the Math. Sciences, Oxford Univ. Press, New York, 1961. MR 25 \#1424.

2. K. F. Clancey and C. R. Putnam, The local spectral behavior of completely sub. normal operators, Trans. Amer. Math. Soc. 163 (1972), 239-244. MR 45 \#934.

3. - The spectra of hyponormal integral operators, Comment. Math. Helv. 46 (1971), 451-456. MR 46 \#31.

4. R. W. Carey and J. D. Pincus, Construction of seminormal operators with prescribed mosaic (preprint).

5. R. G. Douglas, P. S. Muhly and C. Pearcy, Lifting commuting operators, Michigan Math. J. 15 (1968), 385-395. MR 38 \#5046.

6. P. R. Halmos, A Hilbert space problem book, Van Nostrand, Princeton, N. J., 1967. MR 34 \#8178.

7. R. G. Lautzenheiser, Spectral sets, reducing subspaces, and function algebras, Thesis, Indiana Univ., 1973.

8. C. R. Putnam, Commutation properties of Hilbert space operators and related topics, Ergebnisse der Mathematik und ihrer Grenzgebiete, Band 36, Springer-Verlag, New York, 1967. MR 36 \#707. 473-477.

9. - An inequality for the area of hyponormal spectra, Math. Z. 28 (1971),

10. Resolvent vectors, invariant subspaces and sets of zero capacity, Math. Ann. 205 (1973), 165-171. MR 48 \#4772.

11. - Almost normal operators, their spectra and invariant subspaces, Bull. Amer. Math. Soc. 79 (1973), 615-624. MR 49 \#3582.

12. The role of zero sets in the spectra of hyponormal operators, Proc. Amer. Math. Soc. 43 (1974), 137-140. MR 48 \#12130.

13. - Invariant subspaces of certain subnormal operators, J. Functional Analysis 17 (1974), 263-273.

14. F. Riesz and B. Sz.-Nagy, Leçons d'analyse fonctionnelle, Akad. Kiadó, Budapest, 1953; English transl., Functional analysis, Ungar, New York, 1955. MR 15, 132; $17,175$.

15. W. Rudin, Real and complex analysis, McGraw-Hill, New York, 1966. MR 35 $\# 1420$.

16. J. G. Stampfli, Hyponormal operators and spectral density, Trans. Amer. Math. Soc. 117 (1965), 469-476; Errata, ibid. 115 (1965), 550. MR 30 \#3375; 33 \#4686.

17. - A local spectral theory for operators. IV. Invariant subspaces, Indiana Univ. Math. J. 22 (1972/73), 159-167. MR 45 \#5793.

18. Spectral subspaces for hyponormal operators (preprint). 
19. B. Sz.-Nagy and C. Foias, Analyse harmonique des opérateurs de l'espace de Hilbert, Masson, Paris; Akad. Kiadó. Budapest, 1967; English rev. transl., North-Holland, Amsterdam; American Elsevier, New York; Akad. Kiadó, Budapest, 1970. MR 37 \#78; 43 \#947.

20. J. P. Williams, Minimal spectral sets of compact operators, Acta Sci. Math. (Szeged) 28 (1967), 93-106. MR 36 \#725.

DEPARTMENT OF MATHEMATICS, PURDUE UNIVERSITY, WEST LAFAYETTE, INDIANA 47907 